IV. MODELS 


\title{
ROTATIONALLY-ENHANCED STELLAR WINDS \\ (Review Paper)
}

J. M. Marlborough

Department of Astronomy

The University of Western Ontario

London, ON N6A 3K7, Canada

\begin{abstract}
INTRODUCTION
The rotationally-enhanced stellar wind model for Be stars represents one attempt to understand many of the phenomena related to $\mathrm{Be}$ stars in terms of a stellar wind distorted and perhaps also enhanced by rapid stellar rotation. This review will concentrate exclusively on this particular approach; the current status of other attempts to model the circumstellar envelopes (CE) of Be stars are described in other reviews in this volume. It is assumed that Be stars are single stars; if a member of a multiple system, the separation of all components from the $\mathrm{Be}$ star is assumed to be sufficiently large that interactions due to mass transfer from any companion to the Be star are negliglble.

The first suggestion to explain the origin of the emission lines in Be stars was due to Struve (1931; also Struve \& Swings 1932). A thorough discussion of Struve's ideas and their subsequent development, together with other models for the CE which led eventually to the stellar wind models, which are the subject of this review, is contained in Underhill \& Doazan (1982, hereafter UD, p354ff); chapters 10-13 of UD are perhaps the best summary of the Be phenomenon available and should be required reading for all students of Be stars. The first stellar wind models were presented by Doazan (1965), Marlborough (1969) and Hutchings (1970, 1971). These are described in detail by Marlborough (1976, hereafter M1) and also by UD.

In attempting to model the $\mathrm{CE}$ of $\mathrm{Be}$ stars one cannot treat the $C E$ in isolation, since it is part of the atmosphere, the transition zone connecting the interior of the star to the interstellar medium. The basic equations describing the entire structure are given in M1, section 2.1. The discussion there is still relevant to-day; perhaps the only change that needs to be made is the recognition that non-radiative energy effects must be included. Because rotation appears to be important for Be stars, these basic equations are a set of partial differential equations in at least two independent variables, or at least three if a steady state is not assumed. Ideally one hopes to include all relevant physics and solve these equations for the structure and dynamics of the entire atmosphere of a Be star. However despite the wealth of new data which has accumulated recently we are probably no closer to solving these equations than we were ten years ago. To make progress significant simplifications must be made. One approach is to make sufficient simplifying assumptions that a 'theoretical model' containing no arbitrary functions (Castor 1979) can be derived. While such a model
\end{abstract}


may be aesthetically pleasing and may also make definitive predictions, it may also be so idealized as not to apply to any Be star. An alternative approach is to consider 'empirical models' (Castor 1979), also known as ad hoc models, in which parameters such as temperature and velocity for example are arbitrary functions of position and time. This procedure is prima facie less desirable, but nevertheless may be the only way initially of making any progress. It is this latter approach that has been followed largely for stellar wind models of the $\mathrm{CE}$ of $\mathrm{Be}$ stars. Only recently has consideration of theoretical models begun.

\section{EMPIRICAL OR AD HOC STELLAR WIND MODELS}

Because of a lack of knowledge of all the relevant physical effects in the CE of Be stars, the solution of the basic equations has been bypassed in favour of ad hoc models for the dynamical structure. Generally one guesses the velocity, $\vec{u}(\vec{r})$, and the temperature, $T(\vec{r})$, in the $C E$ as functions of position (and time if appropriate) and then obtains the density, $\rho(\vec{r})$, from the equation of continuity. Approximate radiative transfer solutions then yield the ionization-excitation conditions as a function of position. It is of course not necessarily correct that the dynamical structure and the ionization-excitation conditions are uncoupled. The advantages and disadvantages of this approach are well known ( $M 1$, section 3; UD, chp. 11) and will not be repeated here, except to note the general lack of both uniqueness and physical self-consistency of the model so obtained.

The ad hoc stellar wind model, based upon the ideas of Struve (1931), and in some cases also on the ideas of Limber $(1964,1967)$ and Limber \& Marlborough (1968), postulates a nonspherically symmetric distribution, with the CE strongly concentrated to the equatorial plane of the star. Such models are sometimes described as being disklike; in regions where the component of velocity directed away from the rotation axis is small, they resemble the stationary disks of Kogure (1969 and references therein). Perhaps the strongest justification for this ad hoc distribution is the intrinsic linear polarization, numerically small but physically very important, detected in the flux of many Be stars (Coyne \& McLean 1982); this article is a good summary of the importance of polarimetric observations and the conclusions that can be drawn therefrom. If the polarization arises from electron scattering, as seems to be the case, then the region of the $C E$ in which scattering occurs cannot be circularly symmetric about the observer's line of sight when projected onto the plane of the sky. In cases where both the density in the scattering region and the radiation field decrease with increasing distance from the star, a significant fraction of the polarized scattered flux originates near the star (Poeckert \& Marlborough 1978a, hereafter PM; Rudy, 1978). To produce polarization as high as $2 \%$, the necessary departures of the scattering region from spherical symmetry must be large (Haisch \& Cassinelli 1976; Jones 1979). A stellar wind model, in which the $C E$ is strongly concentrated towards the equatorial plane, thus provides naturally a projected distribution which is noncircularly symmetric about the observer's line of sight, except for directions parallel to the rotation axis of the star, and which also contains the required departures from spherical symmetry to produce polarizations 
of order $2 \%$. According to UD, page 357, the ad hoc stellar wind model of PM is "... the most complete Be star model in the number of observable features it is capable of representing." It is thus useful to consider it briefly as an example of an ad hoc, distorted, radiation driven wind model.

The basic dynamical model is discussed in detail in $M 1$ and UD, page $366 \mathrm{ff}$; the determination of the ionization-excitation conditions is discussed by PM. The density distribution is shown in Figure 1 ; the disklike character with density decreasing with increasing distance from the rotation axis is clearly apparent. The comparison between the observed and computed $\mathrm{H} \alpha$ profile and polarization is shown in Figure 2, and the same comparison for the continuous energy distribution and continuum polarization is given in Figure 3 . The mass loss rate, $\dot{m}$, for the $\mathrm{PM}$ model is $4.4 \times 10^{-8} \mathrm{M}_{\odot} \mathrm{y}^{-1}$. Note that not all observational data were obtained simultaneously. Because of the well known variability of Be stars, the importance of obtaining data simultaneously and over as wide a wavelength region as possible cannot be overemphasized. It is useful to recall that the PM model was chosen based only on the comparison of the continuum polarization and the Ho profile with observational data. The favourable agreement of the continuum energy distribution with observations provides some support for the particular model parameters adopted. In retrospect however this agreement may not be unexpected, since to lowest order both $\mathrm{H} \alpha$ and the continuum energy distribution depend in basically the same way on the model properties. Not all comparisons between data and model predictions provided as good agreement however. For example, although the equivalent width of $H \beta$ in Figure 4 is satisfactory, the profile is considerably too broad. One further test involved a comparison of Brackett 14 and Paschen $B$ with infrared data obtained in 1982, much later than the data on which the model was based (Lowe et al. 1985). Although the agreement for Brackett 14 is not too bad, the comparison for Paschen $\beta$ is poor (Figure 5). Whether these disagreements reflect a poor dynamical model and/or problems with the ionization-excitation equilibrium is unknown. The dependence of spectral diagnostics on the various model parameters has been investigated in some detail by Poeckert \& Marlborough (1978b).

Figure 1. Meridional plane density distribution for PM model. Solid lines are density contours $\left(\mathrm{cm}^{-3}\right)$; distances in units of the stellar radius. From PM.

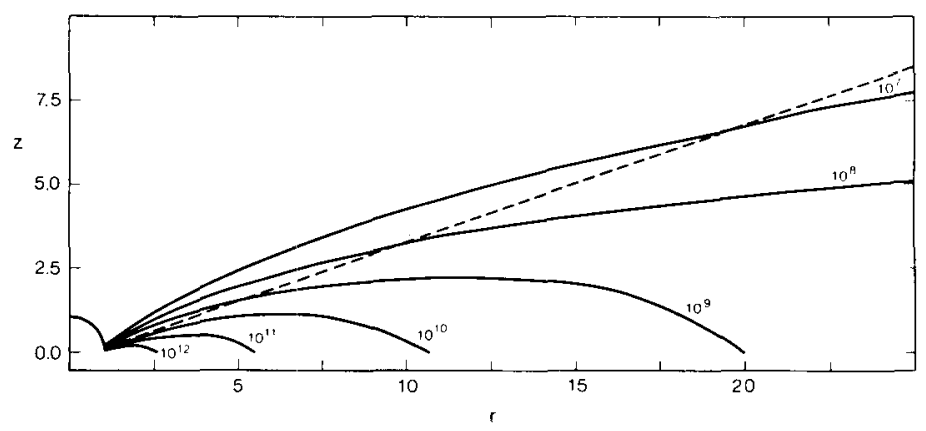


Figure 2. Left: Observed $\mathrm{H} \alpha$ profile and polarization, $\gamma$ Cas. Right: Computed values. Position angle $\psi$ is in degrees. Thin line is observed profile. From PM.
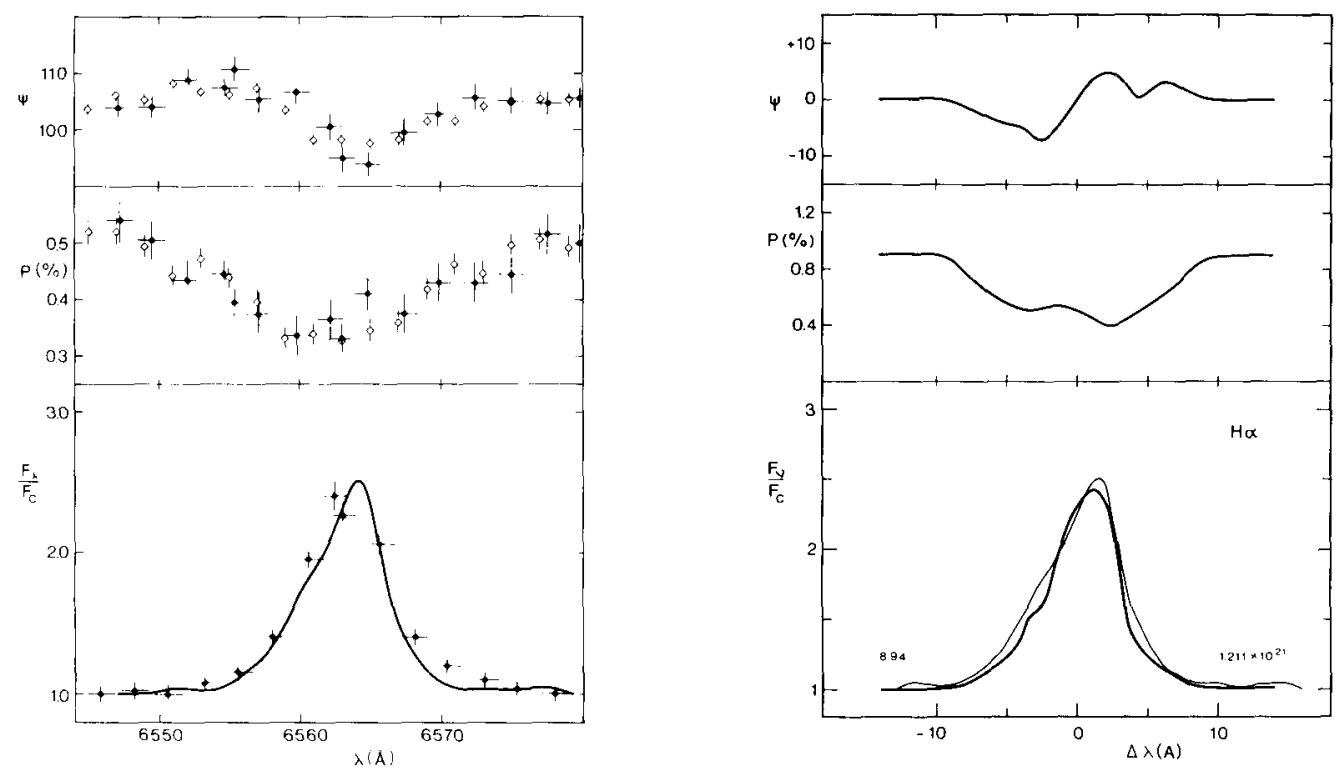

Figure 3. Continuum energy distribution and polarization for the $\gamma$ Cas model. From PM.

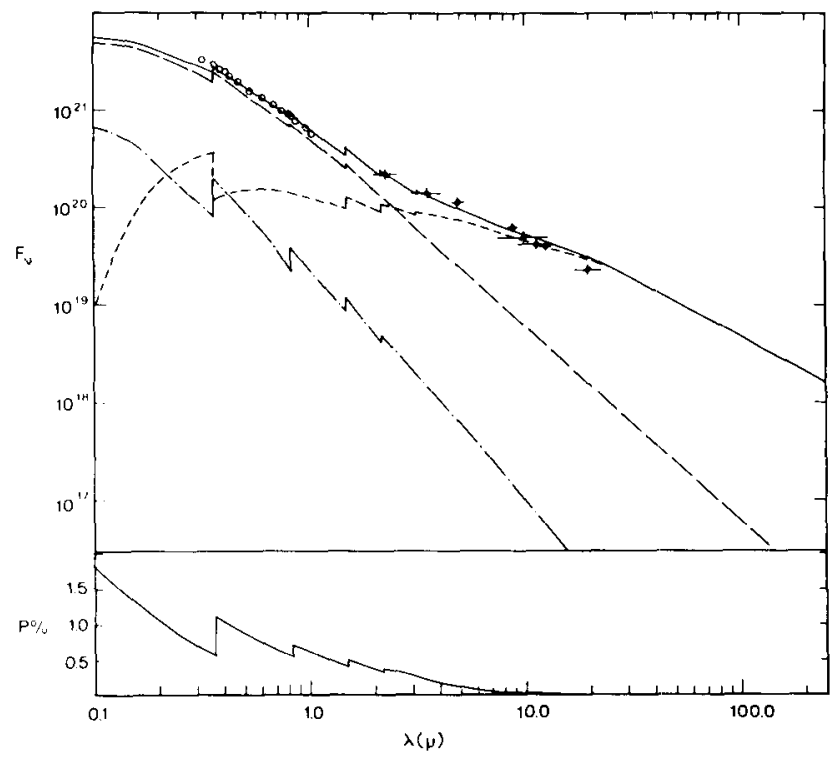


The application of the basic PM model to other stars is described by poeckert (1982 and references therein).

Several recent investigations provide further evidence for the hypothesis that the gas in which Balmer emission and infrared continuum emission arise has a disklike distribution and has motions which are predominantly rotational. Dachs et al. (1986) have studied more than $600 \mathrm{H \alpha}$ line profiles for $55 \mathrm{Be}$ stars. From their analysis they find that for stars of roughly the same $\mathrm{H} \alpha$ equivalent width, there is a good correlation between the full width at half intensity of $\mathrm{H} \alpha$ and $v$ sini of the star. This correlation demonstrates that the $H \alpha$ line is formed in a region where macroscopic motions are predominantly rotational and provides further support for the original ideas of Struve (1931). Their conclusions also support the stellar wind model for the $\mathrm{CE}$, since one expects a gas rotating at moderately large speeds to show considerable departure from spherical symmetry.

Waters (1986) has interpreted IRAS data for 4 Be stars in terms of an equatorial disklike model and has shown that the observations suggest disks which have finite extent and in which the density decreases with increasing distance from the rotation axis faster than inverse square, this faster than inverse square decrease implying a slow acceleration outwards. Inferred values of $\dot{m}$ lie in the range $7 \times 10^{-9}$ to $2 \times 10^{-8} \mathrm{M}_{\Theta} \mathrm{y}^{-1}$. For one star the estimated disk mass is approxima-

Figure 4. Computed polarization and profile for $\mathrm{H} \beta$ for $\gamma \mathrm{Cas}$ model. Observed profile is thin line. From PM.

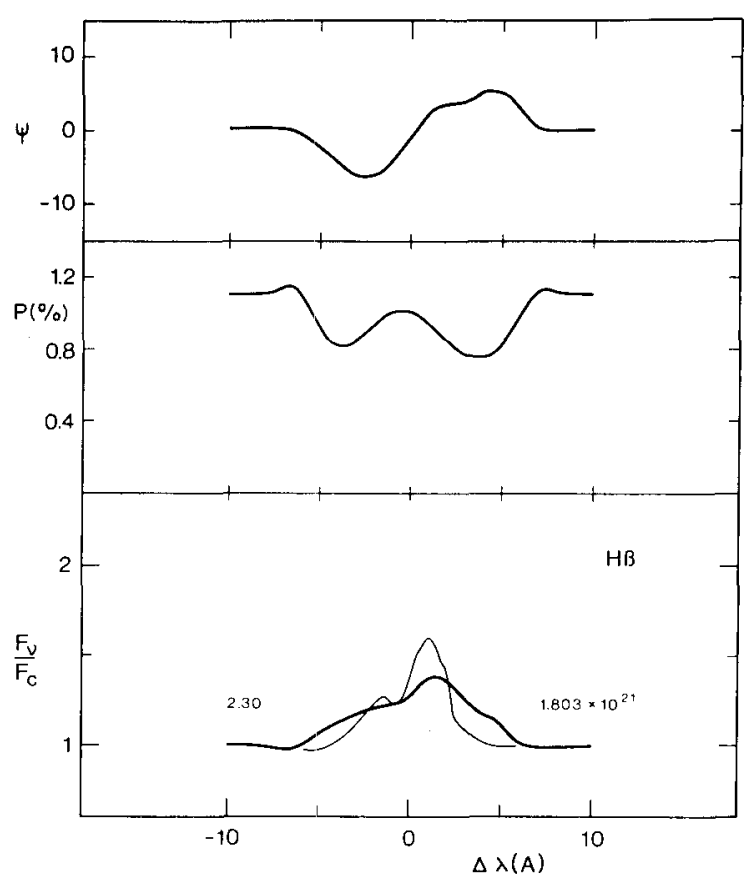


tely $10^{-8} \mathrm{M}_{\odot}$. Neto \& de Freitas Pacheco (1982) have analysed Ho line data and infrared continuum data for a sample of Be stars, using a homogeneous disk model, and deduced total disk masses in the range $10^{-11}$ to $10^{-8} \mathrm{M}_{\odot}$. All these results provide further support for the hypothesis that optical and infrared spectral and photometric data arise in a disklike region concentrated in the equatorial plane of the star, and in which motions are predominantly rotational. The inner region of the ad hoc PM model has exactly this type of structure.

Figure 5. Top: Observed Brackett 14 (solid line) and that predicted by PM model of $\gamma$ Cas. Bottom: Observed Paß (dashed line) and predictions for same model. From Lowe et al. (1985).
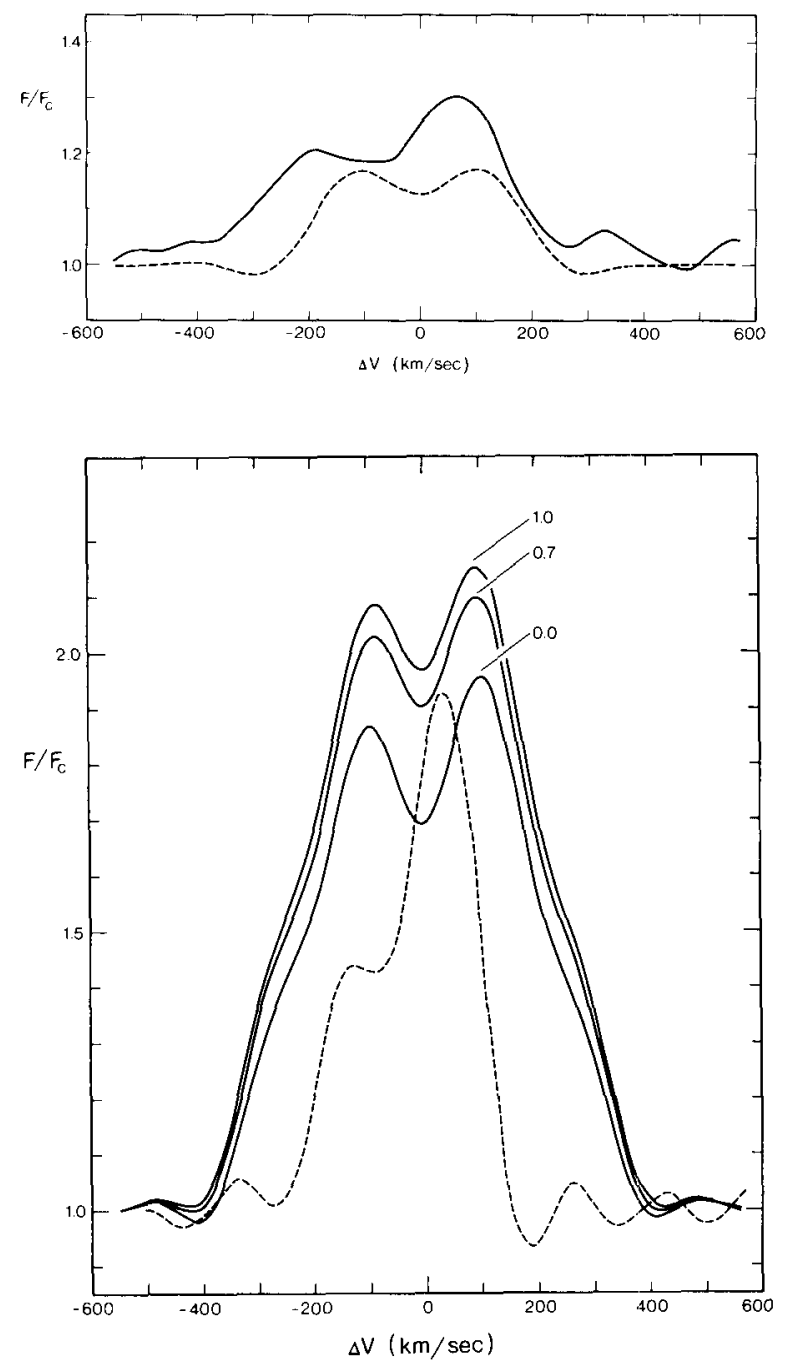
In the past 20 years much additional data has been accumulated about $\mathrm{Be}$ stars especially at ultraviolet (UV) wavelengths. Of particular relevance here is the detection of mass loss at high velocity from observed radial velocities of superionized lines (Marlborough 1982, hereafter M2; UD). Peters (1982) has observed highly shifted, narrow features in broader lines, some of which are superionized, for two $\mathrm{Be}$ stars of moderately low $\mathrm{v}$ sini, implying high velocity material and presumably mass loss at moderately large stellar latitude, if Be stars as a group rotate rapidly. There is at least one fundamental problem to be overcome if one is to understand the existence of superionized ions in the $\mathrm{CE}$ with the presence of matter in lower stages of ionization and/or spectral diagnostics indicating gas of kinetic temperature, $T$, comparable to the effective temperature, $\mathrm{T}$ eff, of the star. Some absorption lines at optical and UV wavelengths are produced by singly and doubly ionized metallic ions. Lamers \& Snow (1978) have shown that $T$ exceeding $10^{5} \mathrm{~K}$ is required in large parts of the wind, if superionized ions arise from collisional ionization; $T$ of order $10^{6} \mathrm{~K}$ is needed somewhere in the $C E$ to produce sufficient soft $x$-rays, if these ions arise by photoionization. In view of both these requirements and the much larger range of radial velocity for superionized features compared to radial velocities from ions in low stages of ionization, it is difficult to understand how the superionized ions can coexist in the same mass elements in which ions of much lower stage of ionization are present. Some separation of the superionized ions from the ions of much lower stage of ionization appears to be needed.

Marlborough et al. (1978) suggested one way in which a region in which superionized ions exist might be added to the PM model. Poeckert (1982) has provided several additional suggestions as to how the low velocity, cool gas in which optical and infrared diagnostics occur might be combined with the region in which superionization takes place. However no quantitative investigation of any of these suggestions has yet been conducted.

Snow (1982a) has estimated $\dot{m}$ for a number of. Be stars from analysis of UV line profiles and found values of $\dot{m}$ ranging from $10^{-11}$ to $3 \times 10^{-9} \mathrm{M}_{\odot} \mathrm{y}^{-1}$, as did de Freitas Pacheco (1982). On average these values of $\dot{m}$ tend to be systematically smaller than $\dot{m}$ values quoted above, which were inferred from optical and/or infrared data. Perhaps these differences are real and simply reflect the fact that $\dot{m}$ at a given time may be a function of position over the surface of the star. Lastly Snow (1982b) has noted that values of $\dot{\mathrm{m}}$ from UV lines for Be stars seem to be what are expected if one extrapolates $\dot{m}$ determined for $O$ and luminous $B$ stars to stars of lower luminosities. Thus a radiation driven stellar wind may have some relevance to the Be phenomenon. In fact Gerasimovic (1934) seems to have been the first to suggest that radiation forces acting selectively on hydrogen through the Lyman $\alpha$ line are critical in the formation of the $\mathrm{CE}$ of Be stars. Recent work on the rotation model has therefore concentrated on radiation driven stellar winds in an attempt to obtain a model or models, which are more self-consistent and perhaps also unique. 
The large ratio of luminosity to mass in hot stars implies that momentum transfer through radiation gas interactions may play an important role in the dynamics of the atmospheres of these stars. Long ago Johnson $(1925,1926)$ and Milne (1926) considered the selective ejection of ions by radiation pressure forces. However it was not until 1970 that Lucy \& Solomon (1970) produced a hydrodynamic model in which the gas is treated as a single fluid. They also noted the problem posed by the presence of $\mathrm{N} \mathrm{V}$, a superionized ion. Collisions between the specific ions on which the radiation force acts and the other constituents of the atmosphere transfer momentum to the remainder of each fluid element and lead to the production of a stellar wind, if the radiation pressure force is sufficiently large. The most complete version of this theory was first introduced by Castor, Abbott \& Klein (1975, hereafter CAK; 1976). Numerous reviews exist. Recent ones which contain a list of major references are Abbott. (1985a) and Lucy (1986). A brief summary of the aspects of the CAK model relevant to the remaining discussion is given here. The above references should be consulted for complete details.

In the original CAK model for a spherically symmetric wind the radiation force arises through the scattering of continuum photons in a very large number of spectral lines. Instead of treating each line in detail CAK parameterized the force as being proportional to $k^{-\alpha}$, where $k$ and $\alpha$ are constants, $k$ being a measure of the number of strong lines and $\alpha$ a measure of the relative number of optically thick and thin lines with $0<\alpha<1$; $t$ is the continuum optical depth of a column whose radial size is the Sobolev length, the distance over which the wind speed increases by the one dimensional thermal speed. Since $t$ involves the velocity gradient, du/dr, the resulting equation of motion is more complex than that encountered in standard solar wind theory because it contains $(\mathrm{du} / \mathrm{dr})^{\alpha}$ and $\alpha$ is non-integral. Nevertheless from consideration of conditions at a regular singular point of the equation, called the critical point, analogous to the sonic point in solar wind theory, a monotonic solution in which the gas accelerates smoothly from low speeds near the star to larger speeds at larger distances can be found. The resulting solution is largely independent of the temperature structure of the wind as long as the temperature does not greatly exceed values consistent with radiative equilibrium. In the original version CAK assumed a pointlike star. This is now known to be a poor approximation, because among other things it significantly overestimates the line acceleration close to the stellar surface, thus leading to a velocity law which is too steep near the star.

The original CAK theory does not provide any explanation for superionization (M2,UD) in hot stars. However it is now recognized from linear stability analyses that the line driven wind solutions are unstable to the development of small amplitude density fluctuations (Hearn 1985; Owocki \& Rybicki 1985; Lucy 1986; and references therein). Although no calculation is yet available for the non-linear development of these instabilities, Lucy (1986 and references therein) has argued that these instabilities will grow and saturate at some finite amplitude, will be driven relative to the background ambient wind, and ultimately produce shock waves, leading to the production of $x$-rays and superionized species throughout the wind by photoionization. 
Despite the apparent success of the radiation driven wind model in accounting for many of the phenomena associated with the winds of hot stars, there are several major problems which must be solved. A non-linear treatment of the instabilities mentioned above must be carried out to test that such instabilities do grow to finite amplitude and survive until they generate shocks as Lucy (1986) has assumed. The general absence of emission components of the UV absorption line profiles in stars on or near the main sequence and including also most of the $\mathrm{Be}$ stars, is troublesome, especially if these lines are formed by pure scattering as is generally assumed. Lastly the presence of discrete components in the absorption part of the UV line profiles has not yet been satisfactorily explained. See Abbott (1985b) for further discussion of these and other problems which must be solved before we can claim to have a reasonable understanding of the winds of hot stars.

4 RADIATION DRIVEN WINDS DISTORTED/ENHANCED BY ROTATION Consider for definiteness a spherical polar coordinate system $(r, \theta, \phi)$ with origin at the centre of the star; $\theta$ is the usual polar angle with $\theta=(0, \pi)$ the rotation axis and $\theta=\pi / 2$ the equatorial plane. The stellar wind has velocity components $\left(u_{r}, u_{\theta}, u_{\phi}\right)$. Even after making many restrictive assumptions such as steady state, axisymmetry, symmetry about the equatorial plane, isotopic gas pressure, inviscid laminar flow, no magnetic field, one is still left with a system of partial differential equations to describe the structure of the wind (Marlborough \& Zamir 1984, hereafter MZ2). Although attempts are underway little progress has yet been made in solving this complicated set of equations.

Many years ago Marlborough \& Zamir (1975, hereafter MZ1), from consideration of the role of rotation in the idealized case of a cool wind driven by continuum radiation forces only (Cassinelli \& Castor 1973), suggested that Be stars might have supersonically expanding stellar winds in regions near their equatorial planes. At essentially the same time however it became clear with the CAK model that the crucial element is momentum transfer in spectral lines rather than solely in the continuum. Barker (1979), Castor (1979), and Abbott (1980) have considered briefly the role of rotation on the structure of a cool wind driven by both line and continuum forces. The potential applicability to Be stars has been discussed in more detail by MZ2. This latter investigation, like most others, separates the flow in the equatorial plane from the three dimensional flow field, of which that in the equatorial plane is a part, by assuming $\partial u_{\theta} / \partial \theta=0, \theta=\pi / 2$; see MZ1 for a brief discussion of this assumption.

Using the original CAK case of a point source star, MZ2 compared semiquantitatively the flow in the equatorial plane to that along the rotation axis, which is described by the spherically symmetric CAK solution. For a wind in which $T<T_{\text {eff }}$, MZ2 predicted that the mass loss rate per unit solid angle $(\dot{m} / 4 \pi)$ will be basically the same at the equator and at the pole. However, even though the critical point for $\theta=\pi / 2$ will be located at a much larger distance from the surface, the wind will have a velocity and acceleration there comparable to those at the critical point for $\theta=0$. An extended disklike region in the equa- 
torial plane therefore develops naturally and the wind attains a lower terminal speed $\left(u_{\infty}\right)$ compared to $u_{\infty}$ in the polar region. A wind of this type might resemble more or less closely the ad hoc stellar wind models of part 2, depending on the density contrast between the regions $\theta=0$ and $\theta=\pi / 2$, i.e. on the stellar rotation speed (urot). This result therefore provides the first dynamical justification for these ad hoc stellar wind models. On the other hand, if $\mathrm{T} \gg \mathrm{T}_{\text {eff }}$, the structure of the wind resembles more closely the spherically symmetric CAK case, even in the equatorial plane, so that less difference between $\rho(r)$ and $u_{r}(r)$ for the regions $\theta=0$ and $\theta=\pi / 2$ is expected.

MZ2 also discussed the problems posed by spectral diagnostics indicating regions of high and low $T$ in the wind, and proposed $a$ plausible steady state model of the $\mathrm{CE}$. In this qualitative model, the regions of the wind near $\theta=\pi / 2$ are denser and have lower velocities $u_{r}$ than regions near $\theta=0$, because the star is rotating rapidly. Superionized ions are assumed to be produced by photoionization due to soft $x$-rays, the $x$-rays arising from shocks in the wind (Lucy 1986 and references therein) and perhaps augmented by a thin corona at the base of the wind (Cassinelli 1985). Apart from the post-shock zones and the corona, if it exists, the wind is generally cool everywhere. Thus superionized ions can exist anywhere in the wind, with the possible exception of the part of the wind near the equatorial plane and close to the star, where the soft $x$-ray optical depth may be too large due to the larger density and/or the wind speed may be too low for shocks to develop. This idea is a modification of scenario 2 of Poeckert (1982). Whether such an ad hoc model will work in practice has not yet been investigated quantitatively.

It is now known that the approximation of a point source star, or equivalently radially streaming radiation, is a poor one even for a spherically symmetric star. CAK first discussed the validity of this approximation and gave the appropriate correction factor for a star of zero limb darkening. Castor (1979) showed that the point source approximation overestimates the line force close to the star and underestimates it at larger distances. Recently Friend \& Abbott (1986, hereafter FA) have redone the original CAK analysis, including a better treatment of the line force and the finite angular size of the star together with the effect of rotation on the flow in the equatorial plane. Inclusion of the finite angular size of the star complicates the resulting equation of motion considerably because the line force term then becomes a function of $r$ and $u_{r}$ in addition to $d u_{r} / d r$.

Compared to the original spherically symmetric CAK model, FA find that the introduction of the finite angular size of the star and the improved line force yield a critical point closer to the star and thus a smaller $\dot{m}$ and a larger $u_{\infty}$. The addition of rotation together with the finite angular size changes predictions considerably with respect to the point source case, because Abbott (1980) has shown that a gradient in the line force, due in this case to the finite disk factor, has a more dominant influence on the location of the critical point than does rotation. Since the location of the critical point is largely determined by the finite angular size factor, it does not move very much as $u_{\text {rot }}$ is increased in contrast to the rotating point source case. Thus since the critical point is approximately fixed, $\dot{m} / 4 \pi$ increases as 


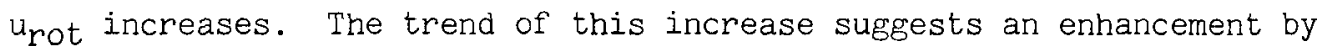
about a factor of 5 in $\dot{m} / 4 \pi$ as breakup speed is approached over that for a similar but nonrotating star. Therefore, just as MZ2 deduced for the case of a point source star, one is led to expect a wind in which the density is increased considerably for regions near $\theta=\pi / 2$ relative to those near $\theta=0$. Since emission per unit volume in Balmer lines and also in optical and infrared continua is roughly proportional to the square of the density, the region of significantly larger density near $\theta=\pi / 2$ is expected to contribute more strongly to this emission than other parts of the wind, thus again simulating the disklike structure of the ad hoc models. Lastly because $\dot{m} / 4 \pi$ is now a function of position on the surface of the star, being larger for $\theta=\pi / 2$ than for $\theta=0$, the model may be able to account in a straightforward manner for the systematic difference between the larger $\dot{m}$ from optical and infrared data compared to the smaller $\dot{m}$ inferred from UV line profiles.

\section{RADIATION DRIVEN WINDS WITH ROTATION AND MAGNETIC FIELDS}

Various authors have suggested that magnetic fields may play

an important role in determining the structure and dynamics of the $C E$ of $\mathrm{Be}$ stars. References are given to older suggestions of this kind in M1 and to more recent ones in MZ2. Here we are concerned solely with large scale magnetic fields, i.e. ordered fields for which a characteristic length scale is of order the stellar radius. Whether such fields are or are not expected to exist is problematical at present. Mestel \& Moss (1977) argued that meridional circulation currents in the interior of a rotating star will tend to confine the magnetic field to the deep interior. This tendency is increased as urot increases so that the fastest rotators, including presumably most Be stars, may have no magnetic field lines emerging from their surfaces, even though their internal magnetic fields could be strong. The status of observational detection of magnetic fields in Be stars has been reviewed in this volume by P. Barker. Typical upper limits to the minimum large scale fields, Which could escape detection by present techniques, are of order $100 \mathrm{~g}$. As emphasized by Barker et al. (1981) however, larger fields of more complex geometry would escape detection by present techniques. There exists also indirect evidence which can be interpreted as implying that highly disordered magnetic fields, consisting of closed magnetic loops separated by regions where the field lines are locally open, may control much of the phenomena observed in 0 and B stars, including Be stars perhaps (Cassinelli 1985). Models involving such disordered fields are discussed in this volume by A. Underhill.

Saito \& Saito (1984) have produced a time dependent, magnetohydrodynamic model for the $C E$ of Be stars. They specificaliy do not include the line radiation force, but they apply their model to a late type $B e$ star for which the neglect of the line radiation force may not be serious. Because of the absence of this force however, their model has little applicability to hotter Be stars.

Poe \& Friend (1986, hereafter PF) have recently produced a model for the structure of a radiation driven wind in the equatorial plane of a star which contains a magnetic field, and applied it to $\mathrm{Be}$ stars. The magnetic field is assumed to be concentrated in the equa- 
torial plane and is treated using the approach of Weber \& Davis (1967). This model of $P F$ can be viewed either as an extension of the point source model of Friend \& MacGregor (1984) to include the finite angular size of the star, or equivalently as the inclusion of an equatorial magnetic field into the model of FA, discussed in part 4. For the point source model of Friend \& MacGregor (1984), the major effect of an increase in the radial component of the magnetic field at the stellar surface $\left(B_{O}\right)$ is to increase the Lorentz force at large radii and thus to increase $u_{\infty}$, i.e. a type of afterburner mechanism. Increasing $u_{r o t}$ for fixed $B_{O}$ increases the centrifugal force and thus raises $\dot{m} / 4 \pi$. Compared to the point source case $\mathrm{PF}$ find a smaller increase in $\dot{\mathrm{m}} / 4 \pi \mathrm{with}$ increasing $u_{r o t}$ at fixed $B_{0}$ and generally a decrease in $u_{\infty}$ with increasing $u_{\text {rot }}$ at fixed $B_{O}$, except for the largest values of $B_{O}$ considered, when the finite angular size of the star is included. Generally $\dot{m} / 4 \pi$ increases with increasing $u_{\text {rot, }}$ independent of $B_{O}$. Again the explanation of this is the same as that for the FA model in part 4. Whether $u_{\infty}$ increases or decreases with increasing urot depends on $B_{0}$. As urot increases the wind accelerates more slowly to lower $u_{\infty}$ at fixed $B_{0}$. At given $u_{r o t}$, larger $B_{O}$ produces a more rapidly accelerating wind, again due to the increased importance of the Lorentz force at large radii. Finally $u_{\phi}$ decreases less rapidly than $r^{-1}$, as either $B_{0}$ or $u_{r o t}$ increase. Although there are differences in detail, the model of $P F$ is qualitatively similar to that of FA. Either provides some dynamical justification for the ad hoc stellar wind models discussed earlier.

\section{APPLICATION TO Be STARS}

To what extent does any of the discussion in parts 2-5 apply to actual Be stars? Here a number of aspects are considered which are pertinent in any attempt to answer this question. Some address directly previously recognized limitations of the rotationally-enhanced stellar wind model for the $\mathrm{CE}$ of Be stars.

\subsection{Radiation Driven Stellar Winds}

Abbott (1979; 1985a, fig. 8) has shown that radiation driven winds are self-initiating in stars more luminous than $\mathrm{M}_{\mathrm{bol}}$ of order -6 . For main sequence stars this limit corresponds to about spectral type B2. Data for normal stars near the main sequence, for which one observes a reasonably sharp division at spectral type B2, separating hotter stars which show spectral evidence for winds and cooler ones which apparently do not, support this prediction (Barker et al. 1984). The UV spectra of Be stars however contain asymmetric and/or displaced absorption lines from superionized ions, indicating winds through the entire B range of spectral subtypes (Barker et al. 1984; Marlborough \& Peters 1986). Therefore some additional mechanism must operate in $\mathrm{Be}$ stars cooler than spectral type B2 to provide the initial acceleration of the wind, after which radiation forces can continue the acceleration to $u_{\infty}$.

The wavelength intervals in which momentum is transferred to the gas by the radiation force are given by Abbott (1985a, fig. 2). For $B$ stars the major contributors coupling gas and radiation are $\mathrm{Fe}$ 
group elements and much of the momentum transfer occurs in the wavelength $(\lambda)$ range $1500<\lambda<3000 \mathrm{~A}$. Severe blending however due to both the high line density and the rapid rotation of the star (M2) make individual lines, which contribute to the radiation force in this $\lambda$ range, largely unrecognizable. It is therefore difficult to test directly the radiation driven wind hypothesis.

Castor (1986) has questioned the applicability of the CAK model to Be stars, especially because of the low values of $h$ obtained from analysis of UV lines. In order to have a radiation driven wind one needs at least one line to be optically thick. For such a situation, $\dot{\mathrm{m}} \simeq \mathrm{L} / \mathrm{c}^{2}$ where $\mathrm{L}$ is the stellar luminosity. This minimum $\dot{\mathrm{m}}$ varies from approximately $10^{-9}$ to $5 \times 10^{-12} \mathrm{M}_{\odot} \mathrm{y}^{-1}$ for main sequence $B$ stars. At the lower end of this range the one fluid approximation may also be inappropriate (Castor et al. 1976, Castor 1986). On the other hand the analysis of optical and infrared data, using either disk or ad hoc stellar wind models, yields values of $\dot{m}$ considerably larger than these necessary lower limits. Likewise the models of FA and PF predict larger mass loss rates for rapidly rotating stars in regions near $\theta=\pi / 2$ compared to those near $\theta=0$. Perhaps therefore Castor's reservation about the application of the radiation driven wind model to Be stars may not present a serious problem.

Thomas (1983) and Doazan \& Thomas (UD, chp. 13) have criticized numerous aspects of the CAK approach. In particular Doazan \& Thomas (UD, chp. 13) ask how the radiation driven wind model can account for differences in $\dot{m}$ between stars of similar spectral type and luminosity class. As one example of this phenomenon relevant to Be stars, they refer to: $\zeta$ Oph $\left(09.5 \mathrm{~V}, \mathrm{v}\right.$ sini $\left.=320 \mathrm{~km} \mathrm{~s}^{-1}\right), \underline{Y}$ Cas $(B O .5 \mathrm{IV}, \mathrm{v}$ $\sin i=230 \mathrm{~km} \mathrm{~s}^{-1}$ ), and $\tau$ SCO (BO V, $\mathrm{V}$ sini $<10 \mathrm{~km} \mathrm{~s}^{-1}$ ); spectral types and $\mathrm{v}$ sini from Slettebak et al. (1975) and Slettebak (1982). The calculations of FA however show that different values of $m / 4 \pi$ for regions near $\theta=\pi / 2$ compared to that for regions near $\theta=0$ occur when both the finite angular size of the star and rotation are included in the original CAK model. Thus it is to be expected that stars of similar spectral type and luminosity class would have steady state, radiation driven winds with different $m$, if the stars had different values of $u_{\text {rot }}$. These three stars certainly satisfy this condition.

Thomas (1983; also Cannon \& Thomas 1977; Doazan \& Thomas in UD, chp. 13) has criticized the CAK assumption of radiative equilibrium or equivalently the neglect of dissipation of energy. Thomas and associates argue partiy by appeal to experiments with trans-sonic flow in laboratory situations that dissipation of some of the energy in the flow will automatically occur, i.e. the gas will be heated, once the flow velocity approaches the one dimensional, thermal speed in the gas, and the flow will then develop a time-dependent character. This timedependent character will persist until the mean flow becomes sufficiently supersonic. Superionized ions will then be formed by collisional ionization close to the stellar photosphere. UV observations clearly show lines from such ions which for many Be stars have their greatest depth near the rest wavelength in the star's reference frame. While it is certainly correct to assert that in laboratory experiments shocks occur frequently in mixed subsonic-supersonic flow, there are some exceptions known. It is also not necessarily obvious that the laboratory 
situation is directly applicable to the stellar case, where flow occurs in a changing gravitational field partly under the action of a large body force from the radiation gas interaction. In fact there may be stellar examples in which a wind passes through a subsonic-supersonic transition without heating. Observational evidence for late-type giants and supergiants (Linsky 1985; Rosner et al. 1985) reveals winds, but as yet does not indicate the presence of either transition region gas $\left(T \simeq 10^{5} \mathrm{~K}\right)$ or $\mathrm{x}$-ray emission $\left(T>10^{6} \mathrm{~K}\right)$, that one would expect following Thomas. For these stars, for which pulsation plays some role in the mass loss process, dissipation of some of the flow energy seems to be either greatly reduced or perhaps even completely eliminated. In the CAK approach the thermal state of the gas does not affect the wind unless the temperature is so high that the gas pressure gradient becomes comparable to the other body forces and/or the degree of ionization changes significantly thus affecting the line radiation force. The radiatively driven instabilities and the shocks assumed to develop subsequently (Lucy 1986 and references therein) are presumed to be such as to give superionized species by photoionization throughout the wind. The ultimate test of this hypothesis is the nonlinear treatment to investigate the growth of these instabilities. The extent to which the radiation driven wind models (FA) agree with data is perhaps the most crucial current test of the CAK approach.

\subsection{Time Variability And Nonradial Pulsation}

Time variability is perhaps the fundamental aspect of the $\mathrm{Be}$ phenomenon. Changes in Balmer emission lines are well known (UD). The UV superionized lines also show dramatic variations (Doazan et al. 1985, 1986). In particular such variations of wind features are observed in Be stars both earlier and later than spectral type B2.

With the exception of the model by Saito \& Saito (1984), all stellar wind models referred to above have been steady state models. They therefore attempt to represent a star in the Be phase. Little consideration has been given to either the transition $B \rightarrow B e$ and the reverse, or to variability within the Be phase. Previous criticisms of the wind models, either ad hoc or otherwise, have emphasized this particular limitation among others. Since the time scale for variations is orders of magnitude shorter than the nuclear time scale it seems reasonable to conclude that changes due to nuclear reactions in the deep interior are not directly responsible for variations in the CE. Following economy of hypotheses, one should at least consider the possibility that whatever process is responsible for the initial acceleration of the wind in stars later than B2, also contributes to the total wind in stars earlier than B2, and perhaps also is responsible for time variations.

In the past few years it has been recognized that nonradial pulsation (NRP) is a common phenomenon in O,B stars. Evidence for NRP, both spectroscopically from line profile variations and/or photometrically from flux changes, has been found in stars from 04 to B6-7, including all luminosity classes and with no restriction to values of $v$ sini (Smith 1986). A detailed discussion of Be stars as nonradial pulsators is given by $D$. Baade in this volume. Of particular relevance to the stellar wind model are the following characteristics of NRP in Be stars: 
(i) modes with latitudinal "quantum number" $1=2$ and modes of higher 1 are simultaneously present, each with amplitudes strongly concentrated toward the equator; (ii) velocity amplitudes are $5-10 \mathrm{~km} \mathrm{~s}^{-1}$ and are strongly radially directed; (iii) temperature fluctuations from wave crest to trough are of order $1000 \mathrm{~K}$ for small 1 modes; and (iv) the particular mode structures are variable in amplitude with time (Smith \& Penrod 1985; Smith 1986).

For several Be stars there is a correlation between a change in the behaviour of NRP and a period of enhanced mass loss as inferred from Balmer emission strength. It is tempting to assume a direct cause and effect relationship. Baade (1985) and Smith \& Penrod (1985) have discussed the potential relevance of these ideas to Be stars. Willson (1986) provides some further thoughts concerning pulsationally driven mass loss, strongly concentrated toward the equatorial plane of a rotating star, and the Be phenomenon. Of particular interest is the possibility that matter so ejected into the CE may remain cool!

If one combines the variable nature of NRP in Be stars with the CAK concept of a radiation driven wind, one may obtain the basic outline for an explanation of the Be phenomenon. What follows here is simply one way in which this might be accomplished. This approach does not deserve to be called a model, at least until quantitative investigations of the suggestions have been considered. For rapidly rotating stars earlier than $\mathrm{B} 2$, a background radiation driven wind may exist as discussed above. The changing character of NRP adds matter to the CE, with greater or less concentration to the equatorial plane depending on the modes present, and in a time varying way. At least close to the star the bulk of the pulsationally ejected matter remains cool. Enhanced periods of line and continuum emission in the optical and infrared thus arise, with the emission strongly concentrated to a disklike region in and near $\theta=\pi / 2$, because such emission is roughly proportional to $\rho^{2}$. Because of this $\rho$ dependence, even modest mass ejection produces dramatic spectral changes at optical and infrared $\lambda$. Variations in linear polarization would be expected to accompany this mass ejection. It is unlikely however that the position angle of polarization would change especially if high 1 modes with azimuthal "quantum number" $\mathrm{m}=1$ are involved. Temperature variations across the disk of the star, from the $I=2$ mode in particular, may provide sufficient modulation of the Lyman continuum radiation field in the $C E$ to account for the $V / R$ variation (UD) of Balmer emission lines (Smith \& Penrod 1985).

It is not clear how the background wind will be affected by this time varying mass ejection, but it seems reasonable to assume it will be affected by some level. Since both large and small mass ejections may occur depending on the interactions between the various modes present, spectral variations at all $\lambda$ accompanying these ejections may be dramatic at some times and less so at others. Perhaps a similar statement will apply to the effect of a particular ejection on the background wind. Presumably the background wind will show broad, asymmetric absorption lines from superionized ions like C IV at all times, the equivalent width of the line depending on $\dot{m}$ and thus on how much hotter than B2 the star of interest is. If some of the pulsationally ejected matter is accelerated to high speeds by the radiation force and some superionization occurs in it, then UV spectral features will also be 
variable. Recall however that in the UV the essential quantity is the column density of a particular absorber as a function of radial velocity and this depends on $\rho$ to the first power. One form this variation might take at some times is that of narrow features moving through the broader underlying absorption line. In the series of observations of $C$ IV in $59 \mathrm{Cyg}$ (B1 V, v sini $=260 \mathrm{~km} \mathrm{~s}^{-1}$ ) presented by Doazan et al. (1985), C IV never disappeared over the 5 year span of the data, although large changes in the equivalent width and profile shape occurred. Whether the above sketch has sufficient potential to account for this dramatic type of variation remains to be seen.

In stars sufficiently cooler than spectral type B2, there is presumably no background radiation driven wind present at all times. Hence the existence of a wind in such stars is probably linked intimately to the changing character of NRP and thus perhaps to phases of enhanced Be emission. For the star $\theta \mathrm{CrB}$ (B6 III, $\mathrm{v}$ sini $=320 \mathrm{~km} \mathrm{~s}^{-1}$ ) the long series of UV data over 5 years (Doazan et al. 1986) shows some

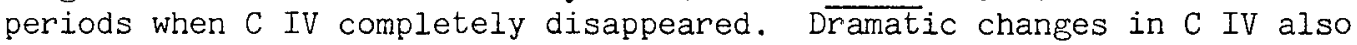
occurred when there was no obvious Balmer emission! This star however may not be a good example of what behaviour to expect in stars cooler than $\mathrm{B} 2$ in general, because its higher luminosity class may put it above the minimum luminosity for a self-initiating radiation driven wind (Abbott 1985a, fig. 8). Barker \& Marlborough (1985) have also noted complex changes in C IV for several Be stars near spectral type B2. Again it remains to be seen whether all these changes can be understood in terms of the sketch outlined here.

\subsection{Emission Components In Lines From Superionized Ions}

In the spectra of most Be stars, lines from superionized ions generally do not have emission components. This same absence of emission components has been noted for $0, B$ stars near the main sequence (Abbott et a1. 1982). The absence of emission components is unexpected if lines are formed by pure scattering. Abbott (1985b) has discussed several possible explanations for the lack of emission components and concluded that no solution, other than photon destruction, seems satisfactory in general.

The calculations of FA and PF indicate however that anisotropic mass loss is expected for a radiation driven wind from a rapid rotator. Therefore for Be stars hotter than B2, anisotropic mass loss is possibly a more realistic explanation than is photon destruction, since the chance of photon destruction in the winds of low $\dot{m}$ characteristic of Be stars is reduced considerably compared to that for $0, B$ stars where $\dot{m}$ is much larger. For Be stars cooler than B2, any wind is expected to be even more anisotropic. Near the star the wind may not even be azimuthally symmetric if NRP is the mechanism responsible for the initial acceleration, because the source of the wind is most likely the peaks of the surface waves. Likewise the extent of the wind in latitude will reflect the latitudinal dependence of the amplitude of the NRP modes producing the mass ejection. Both effects should produce winds which are more anisotropic than those occurring in stars earlier than B2. 


\subsection{Narrow Components}

A common feature of the UV spectra of both O,B and Be stars is the presence of narrow or discrete components, frequently superimposed on a broader, underlying absorption line. These narrow components are normally shifted to shorter $\lambda$ from line centre. In Be stars they are generally found only in the lines of superionized ions. Variability in strength and/or velocity of these narrow components is common (Henrichs 1986 and references therein). There is general agreement that they are formed somewhere within the $\mathrm{CE}$, but where and how they are formed is not yet understood. Abbott (1985b) reviews several suggestions to account for their presence.

Figure 6. Schematic meridional plane structure of the CE. The extent and shape of the cool, disklike region is unknown. It will be influenced by mass ejection accompanying the changing character of NRP. Two possibilities are shown: in the upper the disklike cool region extends to the stellar surface; in the lower a dotted line separates the cool region from a hot zone at the surface. For stars earlier than B2, a background wind arises from all parts of the surface. The arrows indicate the expected variation of wind speed with distance from the star and angle $\theta$. The background wind may be hot or cool with shocks to give superionized ions throughout the wind.

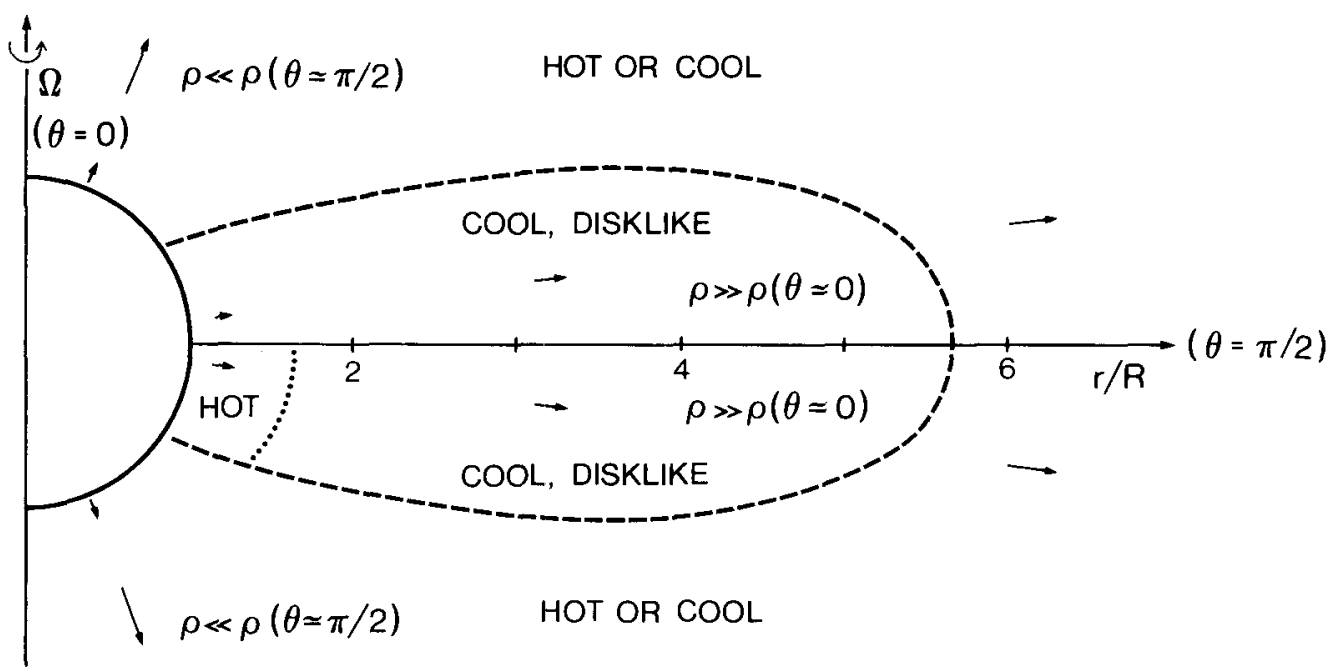


There are at least three Be stars in which changes in these narrow components have been accompanied by changes in Balmer emission and in the character of NRP (Henrichs 1986). This again suggests that NRP may be responsible for much of the variable activity observed in $\mathrm{Be}$ stars.

\section{CONCLUSIONS}

New data discussed in part 2, especially from Dachs et al. (1986) and Waters (1986), strongly support the idea that optical and infrared emission comes from a cool, disklike region concentrated to and extending a few radii from the surface in the equatorial plane. Inclusion of rotation (MZ2) and both the finite angular size of the star and rotation (FA, $P F)$ yield radiation driven winds with larger $\rho(\theta \simeq \pi / 2)$ than $\rho(\theta \simeq 0)$, thus providing dynamical support for the ad hoc wind models. Mass loss near $\theta<<\pi / 2$ may account for displaced UV lines in stars of smaller $v$ sini. Mass loss driven by NRP changes will add to the background wind in stars hotter than B2; in cooler ones the entire CE may arise in this way. Whether the part of the CE outside the cool, disklike zone is hot or cool is still in doubt. A meridional plane section showing the possible arrangement of matter consistent with these ideas is given in Figure 6 .

\section{ACKNOWLEDGEMENTS}

I have benefited greatly from discussions with numerous friends and colleagues including D.C. Abbott, P.K. Barker, V. Doazan, D.B. Friend, L.B. Lucy, A. Slettebak, R.N. Thomas, and M. Zamir. Their comments and suggestions have contributed significantly to my understanding. The errors and misconceptions remaining are exclusively my own.

The preparation of this review was supported by The Natural Sciences and Engineering Research Council of Canada.

REFERENCES

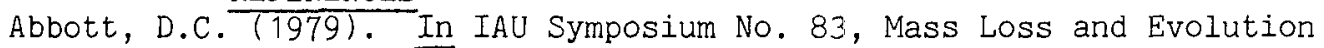
Of O-Type Stars, eds. P. Conti \& C. de Loore, pp. 237-240. Dordrecht: Reidel.

Abbott, D.C. (1980). Ap. J., 242, 1183-1207.

Abbott, D.C. (1985a). In Relations Between Chromospheric-Coronal Heating And Mass Loss In Stars, eds. R. Stalio \& J. Zirker, pp. 265-297. Trieste: Osservatorio Astronomico di Trieste.

Abbott, D.C. (1985b). In Progress In Stellar Spectral Line Formation Theory, eds. J. Beckman \& L. Crivellari, pp. 279-304. Dordrecht: Reidel.

Abbott, D.C., Bohlin, R.C., \& Savage, B.C. (1982). Ap. J. Suppl., 48, 369-383.

Baade, D. (1985). Astr. Ap., 148, 59-66.

Barker, P.K. (1979). Ph. D. Thesis, University of Colorado.

Barker, P.K., Landstreet, J.D., Marlborough, J.M., Thompson, I., \& Maza, J. (1981). Ap. J., 250, 300-305. 
Barker, P.K., Landstreet, J.D. \& Marlborough, J.M. (1984). In Future Of Ultraviolet Astronomy Based On Six Years Of I $\overline{U E}$ Research, eds. J. Mead, R. Chapman, \& Y. Kondo, pp. 219-222. Washington: N.A.S.A. CP-2349.

Barker, P.K. \& Marlborough, J.M. (1985). Ap. J., 288, 329-337. Cannon, C.J. \& Thomas, R.N. (1977). Ap. J., 211, 910-925.

Cassinelli, J.P. (1985). In The Origin Of Nonradiative Heating/Momentum In Hot Stars, eds. A. Underhill \& A. Michalitsianos, pp. 221. Washington: N.A.S.A. CP-2358.

Cassinelli, J.P. \& Castor, J.I. (1973). Ap. J., 179, 189-207.

Castor, J.I. (1979). In IAU Symposium No. 83, Mass Loss And Evolution Of O-Type Stars, eds. P. Conti \& C. de Loore, pp. 175-190. Dordrecht: Reidel.

Castor, J.I. (1986). Pub.A.S.P., 98, 52-55.

Castor, J.I., Abbott, D.C., \& Klein, R.I. (1975). Ap. J., 195, 157-174, (CAK).

Castor, J.I., Abbott, D.C., \& Klein, R.I. (1976). In Physiques Des Mouvements Dans Les Atmospheres Stellaires, eds. R. Cayrel \& M. Steinberg, pp. 364-393. Paris: C.N.R.S.

Coyne, G.V. \& McLean, I.S. (1982). In IAU Symposium No. 98, Be Stars, eds. M. Jaschek \& H. Groth, pp. 77-93. Dordrecht: Reidel.

Dachs, J., Hanuschik, R., Kaiser, D., \& Rohe, D. (1986). Astr. Ap., $159,276-290$.

De Freitas Pacheco, J.A. (1982). M.N.R.A.S., 199, 591-600.

Doazan, V. (1965). Ann. d'Ap., 28, 1-39.

Doazan, V., Grady, C.A., Snow, T.P., Peters, G.J., Marlborough, J.M., Barker, P.K., Bolton, C.T., Bourdonneau, B., Kuhi, L.V., Lyons, R.W., Polidan, R.S., Stalio, R., \& Thomas, R.N. (1985). Astr. Ap., 152, 182-198.

Doazan, V., Marlborough, J.M., Morossi, C., Peters, G.J., Rusconi, L., Sedmak, G., Stalio, R., Thomas, R.N., \& Willis, A. (1986). Astr. Ap., 158, 1-13.

Friend, D.B. \& MacGregor, K.B. (1984). Ap. J., 282, 591-602.

Friend, D.B. \& Abbott, D.C. (1986). Ap. J., submitted, (FA).

Gerasimovic, B.P. (1934). M.N.R.A.S., 94, 737-765.

Haisch, B.M. \& Cassinelli, J.P. (1976). Ap. J., 208, 253-266.

Hearn, A.G. (1985). In The Origin of Nonradiative Heating/Momentum In Hot Stars, eds. A. Underhill \& A. Michalitsianos, pp. 188198. Washington: N.A.S.A. CP-2358.

Henrichs, H. (1986). Pub.A.S.P., 98, 48-51.

Hutchings, J.B. (1970). M.N.R.A.S., 150, 55-66.

Hutchings, J.B. (1971). M.N.R.A.S., $\overline{152}, 109-119$.

Johnson, M.C. (1925). M.N.R.A.S., 85, 813-825.

Johnson, M.C. (1926). M.N.R.A.S., $\overline{86}, 300-319$.

Jones, T.J. (1979). Ap. J., 228, $7 \overline{87}-801$.

Kogure, T. (1969). Astr. Ap. , 1, 253-269.

Lamers, H.J.G.L.M. \& Snow, T.P. (1978). Ap. J., 219, 504-514.

Limber, D.N. (1964). Ap. J., 140, 1391-1400.

Limber, D.N. (1967). Ap. J., 148, 141-157.

Limber, D.N. \& Marlborough, J.M. (1968). Ap. J., 152, 181-193. 
Linsky, J.L. (1985). In Relations Between Chromospheric-Coronal Heating And Mass Loss In Stars, eds. R. Stalio \& J. Zirker, pp.5589. Trieste: Osservatorio Astronomico di Trieste.

Lowe, R.P., Moorhead, J.M., Wehlau, W.H., Barker, P.K., \& Marlborough, J.M. (1985). Ap. J., 290, 325-336.

Lucy, L.B. (1986). In IAU Colloquium No. 89, Radiation Hydrodynamics In Stars And Compact Objects, in press.

Lucy, L.B. \& Solomon, P.M. (1970). Ap. J., 159, 879-893. Marlborough, J.M. (1969). Ap. J., 156, 135-155.

Marlborough, J.M. (1976). In IAU Symposium No. 70, Be And Shell Stars, ed. A. Slettebak, pp. 335-370. Dordrecht: Reidel, (M1).

Marlborough, J.M. (1982). In IAU Symposium No. 98, Be Stars, eds. M. Jaschek \& H. Groth, pp. 361-376. Dordrecht: Reidel, (M2) .

Marlborough, J.M. \& Zamir, M. (1975), Ap. J., 195, 145-155, (MZ1). Marlborough, J.M., Snow, T.P., \& Slettebak, A. (1978). Ap. J., 224, $157-166$.

Marlborough, J.M. \& Zamir, M. (1984). Ap. J. 276, 706-723, (MZ2). Marlborough, J.M. \& Peters, G.J. (1986), Ap. J. Suppl., in press. Mestel, L. \& Moss, D.L. (1977). M.N.R.A.S., 178, 27-49. Milne, E.A. (1926). M.N.R.A.S., 86, 459-473.

Neto, A.D. \& de Freitas Pacheco, J.A. (1982). M.N.R.A.S., 198, 659-668. Owocki, S.P. \& Rybicki, G.B. (1985). Ap. J., 299, 265-276.

Peters, G.J. (1982). Ap. J. Letters, 253, L33-L37.

Poe, C.H. \& Friend, D.B. (1986). Ap. J., in press, (PF).

Poeckert, R. (1982). In IAU Symposium No. 98, Be Stars, eds. M. Jaschek \& H. Groth, pp. 453-483. Dordrecht: Reidel.

Poeckert, R. \& Marlborough, J.M. (1978a). Ap. J., 220, 940-961, (PM) . Poeckert, R. \& Marlborough, J.M. (1978b). Ap. J. Suppl., 38, 229-252. Rosner, R., Golub, L., \& Vaiana, G.S. (1985). Ann. Rev. Astr. Ap., 23, 413-452.

Rudy, R.J. (1978). Pub.A.S.P., 90, 688-691.

Saito, M. \& Saito, Y. (1984). Pub.A.S.J., 36, 603-612.

Slettebak, A. (1982). Ap. J. Suppl., 50, 55-84.

Slet.tebak, A., Collins, G.W., Boyce, P.B., White, N.M., \& Parkinson, T.D. (1975). Ap. J. Suppl., 29, 137-159.

Smith, M.A. (1986). Pub.A.S.P., 98, 33-34.

Smith, M.A. \& Penrod, G.D. (1985). In Relations Between ChromosphericCoronal Heating and Mass Loss In Stars, eds. R. Stalio \&

J. Zirker, pp. 394-404. Trieste: Osservatorio Astronomico di Trieste.

Snow, T.P. (1982a). In IAU Symposium No. 98, Be Stars, eds. M. Jaschek \& H. Groth, pp. 377-385. Dordrecht: Reidel.

Snow, T.P. (1982b). Ap. J. Letters, 253, L39-L42.

Struve, O. (1931). Ap. J., 73, 94-103.

Struve, 0. \& Swings, P. (1932). Ap. J., 75, 161-184.

Thomas, R.N. (1983). Stellar Atmospheric Structural Patterns.

Washington: N.A.S.A. SP-471.

Underhill, A. \& Doazan, V. (1982). B Stars With And Without Emission

Lines. Washington: N.A.S.A. SP-465, (UD).

Waters, L.B.F.M. (1986). Astr. Ap., in press.

Weber, E.J. \& Davis, L. (1967). Ap. J., 148, 217-227.

Willson, L.A. (1986). Pub.A.S.P., 98, 37 $\overline{-40}$. 


\section{DISCUSSION FOLLOWING MARLBOROUGH}

Smith (M.A.):

I wish to comment on your "big if" concerning reports of changes in Be activity being correlated with changes in nonradial pulsation parameters. These reports are in the main attributable to Dan Penrod, who unfortunately could not be here to defend his work, and so I will try: Don's reports are based on extensive spectroscopic observations of a number of Be stars. These correlations are based on gross properties of the line profile, i.e. changes in the amplitudes of line width and asymmetry. These properties are not to be confused with what may seem to many as fuzzy small-amplitude bumps travelling across the line profile. One doesn't need fancy instrumentation to observe the correlations in these line profile parameters; they are tied closely to the amplitude variations in radial velocity and light. For my part, I have followed $\lambda$ Eri (B2e) for two observing seasons, and I am certainly able to confirm Don's point that the level of Be emission is anticorrelated with the amplitude of an alleged nonradial $l=2$ mode. As to the correctness of the pulsation model itself, one of the most convincing arguments in favor is that for certain midly-active Be stars, one observes variable $\mathrm{V} / \mathrm{R}$ emission in the wings of $\mathrm{HeI}$ lines which is correlated with the period. It is easiest to understand these rapid and correlated emission changes in terms of two "searchlights" illuminating a circumstellar disk which are located $180^{\circ}$ apart on the star's equator. This consideration suggests an $m=l=2$ nonradial mode. To be sure, it is possible to construct models with hot spots on the star's surface, but they can only work under contrived circumstances and only for certain stars. In fact, if one chooses any touted competing model to nonradial pulsations, one can invariably find an example where the profile or $\mathrm{V} / \mathrm{R}$ period can't be matched to the rotation period. Pulsation models do not suffer this disadvantage.

Thomas:

My primary objection centers on lack of any mechanism to produce the variability in far UV lines for $300 \mathrm{kms}^{-1}-200 \mathrm{kms}^{-1}$ in some stars.

I do not see how a photospheric radiation field accelerating the flow can do this. I do see how a coronal-chromospheric radiation field of variable strength can do this, if one attributes such variation primarily to variations of opacity of the chromosphere-corona. Such opacity variation comes directly from a variation in mass output at the photospheric level. Variations in the mass output can cause variations in the density in the chromospherecorona, and variations in velocity in the photosphere. A multitude of combined far UV and visual observations show variable photospheric mass outflow to characterize Be stars.

An equally strong objection comes from the aerodynamic experience that major variability lies in the transonic region, not the supersonic, if one looks at a flow that begins at low subsonic velocity, goes through transonic transition, and ends as a large supersonic flow.

On this basis, we predicted in 1973 that any star showing a mass outflow will produce a low-enough lying chromosphere-corona that absorption, not emission, superionization phenomena will occur. We predicted the chromosphere-corona to occur at $r / R_{*}<1.01$.

I strongly disagree with your belief that the Lucy and Soloman, Castor-AbbottKlein etc. photospheric, steady-state radiative acceleration origin model is a reasonable first approximation to modeling Be stars. My primary objection is that such a theory a priori excludes the possibility of modelling the strongest feature of Be stars - strong variability in velocities observed in the far UV in some bright hot Be stars for $200-300 \mathrm{kms}^{-1}$. My equally strong opinion is that the theory has no nonradiative heating. Therefore it cannot produce those coronae whose variable radiative acceleration can produce the observed far 
UV variations. Also, the theory cannot produce those superionized lines in Be and B stars whose observation gives the evidence for velocities.

Baade:

$\mathrm{H} \alpha$ profiles of non-emission line B stars (Furenlid and Young 1980) show for moderate to large vsini's blue absorption whose strength clearly correlates with vsini. As these stars are otherwise inconspicuous this observation probably confirms that rotation leads to an equatorial density enhancement of the wind.

Since $\mathrm{Be}$ stars do not rotate more rapidly than $\mathrm{Bn}$ stars and the mass loss rates of Be stars are highly variable the explanation of Be stars cannot only be rotation as is well known. But the mere addition of nonradial pulsations is not sufficient either because there is no correlation between instantaneous mass loss rate and instantaneous pulsation amplitude.

The long-term correlation between these two quantities, as established by Penrod, does however suggest that nonradial pulsations are involved in causing mass loss. This means that in addition to rapid rotation and a low-order nonradial pulsation mode some other conditions must be fulfilled in order to let the pulsation become efficient in causing mass loss. One possibility is that the pulsations themselves slowly build up this condition in which case the process may be a self-regulating relaxation cycle of both rotation and pulsation.

Dachs:

In your basic Poeckert-Marlborough model, you are only considering and fitting asymmetric $\mathrm{H} \alpha$ emission line profiles which may be called "red-dominated", indicating expansion through the disk. Be star observations show that, if $\mathrm{H} \alpha$ emission has some asymmetry, in $50 \%$ of the cases the asymmetry is of the "blue-dominated" type. Can you also explain blue-dominated emission-line profiles?

Marlborough:

In the extensive set of calculations for the Poeckert- Marlborough model some profiles, more blue than red dominated, occurred for the disk-like model viewed at moderately low inclination angles. However, for cases where a given star of moderately large vsini alternates from red dominated to blue dominated, the only way to account for the blue dominated phase would seem to require reversing the sign of the radial velocity component. Needless to say, such a procedure is not consistent with a steady state, stellar wind model.

Baade:

Averaged over $\sim 1$ day, the effects of the pulsations on the disk is axisymmetric. How would such a process lead to asymmetric variations of the disk on a timescale of years?

Marlborough:

The V/R variations occurring on a timescale of years must arise from some other effect. Perhaps some Be stars in this group are members of binary systems which may or may not be interacting binaries. In either case the gravitational effect of the companion could affect significantly both the geometry and dynamics of the circumstellar matter around the Be star.

Stalio:

The ratio of mass loss at the equator to mass loss at the poles has been calculated for rotational velocity approaching critical velocity. What is this ratio if the star rotates at $\mathrm{v}_{\text {rot }}<0.85 \mathrm{v}_{\text {crit }}$ ? 
Marlborough:

For each of several different values of the strength of the radial magnetic field at the surface of the star, Poe and Friend calculated mass loss rates in the equatorial plane for a range of rotational velocities, the maximum being approximately 0.85 of the critical value. For the maximum rotation speed considered the increase in mass loss rate relative to a non-rotating star was about a factor of 5-10. At lower rotation speeds the enhancement in mass loss rate is smaller, the mass loss rate in the equatorial plane varying nonlinearly with rotation speed. The specific details are given in their paper.

Friend:

The equatorial mass loss enhancement of 5 is for a rotational velocity of about $85 \%$ of the critical value. If the rotational velocity is increased further, the enhancement appears to go to infinity as the critical value is approached.

Underhill:

You and your questioners have indicated ways in which to concentrate mechanical energy in certain dense layers of the model, even in 3-dimensional volumes. However, what you observe is the result of energy being added to or removed from local volumes of low-density plasma. How does one transfer energy from the source area to the radiating volume?

Marlborough:

There are many possible mechanisms: electromagnetic radiation, dissipation of kinetic energy in macroscopic motions, acoustic waves, magnetohydrodynamic waves, magnetic field line reconnection, etc. Which of these are more important is currently unknown, I think. Differences from star to star might also be expected.

Kogure:

As for the phase change $\mathrm{B}$ to Be you have mentioned nonradial pulsations as a possible cause of such changes. From the viewpoint of spectroscopic study, I would emphasize the role of vertical extension of disk-like envelopes. Some Be stars, particularly among shell stars, show evidence to have very vertically thin envelopes, whereas normal Be stars may have sufficiently vertically expanded envelopes. In my view, vertical extension may be an important parameter distinguishing $\mathrm{Be}$ and shell stars. 